\title{
EMBEDDINGS OF LOCALLY FINITE METRIC SPACES INTO BANACH SPACES
}

\author{
F. BAUDIER AND G. LANCIEN
}

(Communicated by N. Tomczak-Jaegermann)

\begin{abstract}
We show that if $X$ is a Banach space without cotype, then every locally finite metric space embeds metrically into $X$.
\end{abstract}

\section{INTRODUCTION}

Let $X$ and $Y$ be two Banach spaces. If $X$ and $Y$ are linearly isomorphic, the Banach-Mazur distance between $X$ and $Y$, denoted by $d_{B M}(X, Y)$, is the infimum of $\|T\|\left\|T^{-1}\right\|$, over all linear isomorphisms $T$ from $X$ onto $Y$.

For $p \in[1, \infty]$ and $n \in \mathbb{N}, \ell_{p}^{n}$ denotes the space $\mathbb{R}^{n}$ equipped with the $\ell_{p}$ norm. We say that a Banach space $X$ uniformly contains the $\ell_{p}^{n}$ 's if there is a constant $C \geq 1$ such that for every integer $n, X$ admits an $n$-dimensional subspace $Y$ so that $d_{B M}\left(\ell_{p}^{n}, Y\right) \leq C$.

A metric space $M$ is locally finite if any ball of $M$ with finite radius is finite. If moreover, there is a function $C:(0,+\infty) \rightarrow \mathbb{N}$ such that any ball of radius $r$ contains at most $C(r)$ points, we say that $M$ has a bounded geometry.

Let $(M, d)$ and $(N, \delta)$ be two metric spaces and $f: M \rightarrow N$ be a map. For $t>0$ define

$$
\rho_{f}(t)=\inf \{\delta(f(x), f(y)), \quad d(x, y) \geq t\}
$$

and

$$
\omega_{f}(t)=\sup \{\delta(f(x), f(y)), \quad d(x, y) \leq t\} .
$$

We say that $f$ is a coarse embedding if $\omega_{f}(t)$ is finite for all $t>0$ and $\lim _{t \rightarrow \infty} \rho_{f}(t)=$ $\infty$.

Suppose now that $f$ is injective. We say that $f$ is a uniform embedding if $f$ and $f^{-1}$ are uniformly continuous. Following [7], we also define the distortion of $f$ to be

$$
\operatorname{dist}(f):=\|f\|_{L i p}\left\|f^{-1}\right\|_{\text {Lip }}=\sup _{x \neq y \in M} \frac{\delta(f(x), f(y))}{d(x, y)} . \sup _{x \neq y \in M} \frac{d(x, y)}{\delta(f(x), f(y))} .
$$

If the distortion of $f$ is finite, we say that $f$ is a metric embedding and that $M$ metrically embeds into $N$ and we denote $M \hookrightarrow N$. If $\operatorname{dist}(f) \leq C$, we use the notation $M \stackrel{C}{\hookrightarrow} N$.

Received by the editors October 4, 2006 and, in revised form, January 10, 2007.

2000 Mathematics Subject Classification. Primary 46B25; Secondary 51F99. 
A celebrated result of I. Aharoni [1] asserts that every separable metric space metrically embeds into $c_{0}$ (the space of all real sequences converging to 0 , equipped with the supremum norm). It is an open problem to know what are the Banach spaces that share this property with $c_{0}$. In other words, if $c_{0}$ metrically embeds into a separable Banach space $X$, do we have that $X$ contains a closed subspace which is linearly isomorphic to $c_{0}$ ? Very recently, N.J. Kalton [3] made an important step in this direction by showing that one of the iterated duals of such a Banach space has to be nonseparable (in particular, $X$ cannot be reflexive). In fact, he even proved it for $X$ such that $c_{0}$ coarsely or uniformly embeds into $X$.

In another direction, N. Brown and E. Guentner proved in [2] that any metric space with bounded geometry coarsely embeds into a reflexive Banach space. This was also improved by Kalton in [3], who obtained that any locally finite metric space uniformly and coarsely embeds into a reflexive space. These results are connected with the coarse Novikov conjecture. Indeed, Kasparov and Yu have shown in [4] that if a metric space with bounded geometry coarsely embeds into a super-reflexive Banach space, then it satisfies this important conjecture. In this paper, we improve the results of Brown, Guentner and Kalton, by showing that any Banach space uniformly containing the $\ell_{\infty}^{n}$ 's is metrically universal for all locally finite metric spaces. It should also be noted that Brown and Guentner used a specific space, $\left(\sum \ell_{p_{n}}\right)_{2}$, with $p_{n}$ tending to infinity and that this space fulfils our hypothesis.

\section{Results}

Theorem 2.1. There exists a universal constant $C>1$ such that for every Banach space $X$ uniformly containing the $\ell_{\infty}^{n}$ 's and every locally finite metric space $(M, d)$, $M \stackrel{C}{\hookrightarrow} X$.

Proof. Let $X$ be a Banach space uniformly containing the $\ell_{\infty}^{n}$ 's (or equivalently without any nontrivial cotype). Our first lemma follows directly from the classical work of B. Maurey and G. Pisier [6].

Lemma 2.2. For any finite codimensional subspace $Y$ of $X$, any $\varepsilon>0$ and any $n \in \mathbb{N}$, there exists a subspace $F$ of $Y$ such that $d_{B M}\left(\ell_{\infty}^{n}, F\right)<1+\varepsilon$.

We shall also need the following version of Mazur's Lemma (see for instance [5], page 4 , for a proof).

Lemma 2.3. Let $X$ be an infinite dimensional Banach space, $E$ be a finite dimensional subspace of $X$ and $\varepsilon>0$. Then there is a finite codimensional subspace $Y$ of $X$ such that:

$$
\forall x \in E \quad \forall y \in Y, \quad\|x\| \leq(1+\varepsilon)\|x+y\| .
$$

Now consider a locally finite metric space $(M, d)$. For $t \in M$ and $r \geq 0, B(t, r)=$ $\{s \in M, d(s, t) \leq r\}$. We fix a point $t_{0}$ in $M$ and denote $|t|=d\left(t, t_{0}\right)$, for $t \in M$. Since $M$ is locally finite, we may assume, by multiplying $d$ by a constant if necessary, that $B\left(t_{0}, 1\right)=\left\{t_{0}\right\}$. For any nonnegative integer $n$, we denote $B_{n}=B\left(t_{0}, 2^{n+1}\right)$. Using Lemmas 2.2 and 2.3, together with the fact that each ball $B_{n}$ is finite, we can build inductively finite dimensional subspaces $\left(F_{n}\right)_{n=0}^{\infty}$ of $X$ and $\left(T_{n}\right)_{n=0}^{\infty}$ so that for every $n \geq 0, T_{n}$ is a linear isomorphism from $\ell_{\infty}\left(B_{n}\right)$ onto $F_{n}$ satisfying

$$
\forall u \in \ell_{\infty}\left(B_{n}\right), \quad \frac{1}{2}\|u\| \leq\left\|T_{n} u\right\| \leq\|u\|
$$


and also such that $\left(F_{n}\right)_{n=0}^{\infty}$ is a Schauder finite dimensional decomposition of its closed linear span $Z$. More precisely, if $P_{n}$ is the projection from $Z$ onto $F_{0} \oplus \ldots \oplus F_{n}$ with kernel $\overline{\mathrm{sp}}\left(\bigcup_{i=n+1}^{\infty} F_{i}\right)$, we will assume, as we may, that $\left\|P_{n}\right\| \leq 2$. We now denote $\Pi_{0}=P_{0}$ and $\Pi_{n}=P_{n}-P_{n-1}$ for $n \geq 1$. We have that $\left\|\Pi_{n}\right\| \leq 4$.

We now consider $\varphi_{n}: B_{n} \rightarrow \ell_{\infty}\left(B_{n}\right)$ defined by

$$
\forall t \in B_{n}, \quad \varphi_{n}(t)=\left(d(s, t)-d\left(s, t_{0}\right)\right)_{s \in B_{n}}=(d(s, t)-|s|)_{s \in B_{n}} .
$$

The map $\varphi_{n}$ is clearly an isometric embedding of $B_{n}$ into $\ell_{\infty}\left(B_{n}\right)$. Then we set:

$$
\forall t \in B_{n}, \quad f_{n}(t)=T_{n}\left(\varphi_{n}(t)\right) \in F_{n} .
$$

Finally we construct a map $f: M \rightarrow X$ as follows:

(i) $f\left(t_{0}\right)=0$.

(ii) For $n \geq 0$ and $2^{n} \leq|t|<2^{n+1}$ :

$$
f(t)=\lambda f_{n}(t)+(1-\lambda) f_{n+1}(t), \text { where } \lambda=\frac{2^{n+1}-|t|}{2^{n}} .
$$

We will show that $\operatorname{dist}(f) \leq 9 \times 24=216$.

Note first that for any $t$ in $M$,

$$
\frac{1}{16}|t| \leq\|f(t)\| \leq|t| .
$$

We start by showing that $f$ is Lipschitz. Let $t, t^{\prime} \in M \backslash\left\{t_{0}\right\}$ and assume, as we may, that $1 \leq|t| \leq\left|t^{\prime}\right|$ (we recall that $B\left(t_{0}, 1\right)=\left\{t_{0}\right\}$ ).

I) If $|t| \leq \frac{1}{2}\left|t^{\prime}\right|$, then

$$
\left\|f(t)-f\left(t^{\prime}\right)\right\| \leq|t|+\left|t^{\prime}\right| \leq \frac{3}{2}\left|t^{\prime}\right| \leq 3\left(\left|t^{\prime}\right|-|t|\right) \leq 3 d\left(t, t^{\prime}\right) .
$$

II) If $\frac{1}{2}\left|t^{\prime}\right|<|t| \leq\left|t^{\prime}\right|$, we have two different cases to consider.

1) $2^{n} \leq|t| \leq\left|t^{\prime}\right|<2^{n+1}$, for some $n \geq 0$. Then, let

$$
\lambda=\frac{2^{n+1}-|t|}{2^{n}} \text { and } \lambda^{\prime}=\frac{2^{n+1}-\left|t^{\prime}\right|}{2^{n}} .
$$

We have that

$$
\left|\lambda-\lambda^{\prime}\right|=\frac{\left|t^{\prime}\right|-|t|}{2^{n}} \leq \frac{d\left(t, t^{\prime}\right)}{2^{n}}
$$

SO

$$
\begin{aligned}
\left\|f(t)-f\left(t^{\prime}\right)\right\| & =\left\|\lambda f_{n}(t)-\lambda^{\prime} f_{n}\left(t^{\prime}\right)+(1-\lambda) f_{n+1}(t)-\left(1-\lambda^{\prime}\right) f_{n+1}\left(t^{\prime}\right)\right\| \\
& \leq \lambda\left\|f_{n}(t)-f_{n}\left(t^{\prime}\right)\right\|+(1-\lambda)\left\|f_{n+1}(t)-f_{n+1}\left(t^{\prime}\right)\right\|+2\left|\lambda-\lambda^{\prime}\right|\left|t^{\prime}\right| \\
& \leq d\left(t, t^{\prime}\right)+2^{n+2}\left|\lambda-\lambda^{\prime}\right| \leq 5 d\left(t, t^{\prime}\right) .
\end{aligned}
$$

2) $2^{n} \leq|t|<2^{n+1} \leq\left|t^{\prime}\right|<2^{n+2}$, for some $n \geq 0$. Then, let

$$
\lambda=\frac{2^{n+1}-|t|}{2^{n}} \text { and } \lambda^{\prime}=\frac{2^{n+2}-\left|t^{\prime}\right|}{2^{n+1}} .
$$

We have that

$$
\lambda \leq \frac{d\left(t, t^{\prime}\right)}{2^{n}}, \text { so } \lambda|t| \leq 2 d\left(t, t^{\prime}\right)
$$


Similarly

$$
\begin{gathered}
1-\lambda^{\prime}=\frac{\left|t^{\prime}\right|-2^{n+1}}{2^{n+1}} \leq \frac{d\left(t, t^{\prime}\right)}{2^{n+1}} \text { and }\left(1-\lambda^{\prime}\right)\left|t^{\prime}\right| \leq 2 d\left(t, t^{\prime}\right) . \\
\left\|f(t)-f\left(t^{\prime}\right)\right\|=\left\|\lambda f_{n}(t)+(1-\lambda) f_{n+1}(t)-\lambda^{\prime} f_{n+1}\left(t^{\prime}\right)-\left(1-\lambda^{\prime}\right) f_{n+2}\left(t^{\prime}\right)\right\| \\
\leq \lambda\left(\left\|f_{n}(t)\right\|+\left\|f_{n+1}(t)\right\|\right)+\left(1-\lambda^{\prime}\right)\left(\left\|f_{n+1}\left(t^{\prime}\right)\right\|+\left\|f_{n+2}\left(t^{\prime}\right)\right\|\right) \\
\quad+\left\|f_{n+1}(t)-f_{n+1}\left(t^{\prime}\right)\right\| \\
\leq d\left(t, t^{\prime}\right)+2 \lambda|t|+2\left(1-\lambda^{\prime}\right)\left|t^{\prime}\right| \leq 9 d\left(t, t^{\prime}\right) .
\end{gathered}
$$

We have shown that $f$ is 9-Lipschitz.

We shall now prove that $f^{-1}$ is Lipschitz. We consider $t, t^{\prime} \in M \backslash\left\{t_{0}\right\}$ and assume again that $1 \leq|t| \leq\left|t^{\prime}\right|$. We need to study three different cases. In our discussion, whenever $|t|$ (respectively $\left|t^{\prime}\right|$ ) will belong to $\left[2^{m}, 2^{m+1}\right.$ ), for some integer $m$, we shall denote

$$
\lambda=\frac{2^{m+1}-|t|}{2^{m}} \quad\left(\text { respectively } \quad \lambda^{\prime}=\frac{2^{m+1}-\left|t^{\prime}\right|}{2^{m}}\right) .
$$

1) $2^{n} \leq|t| \leq\left|t^{\prime}\right|<2^{n+1}$, for some $n \geq 0$. Then

$$
\begin{aligned}
& \Pi_{n}\left(f(t)-f\left(t^{\prime}\right)\right)=T_{n}\left(\lambda \varphi_{n}(t)-\lambda^{\prime} \varphi_{n}\left(t^{\prime}\right)\right) \text { and } \\
& {\left[\lambda \varphi_{n}(t)-\lambda^{\prime} \varphi_{n}\left(t^{\prime}\right)\right]_{\left.\right|_{s=t^{\prime}}}=\lambda d\left(t, t^{\prime}\right)+\left(\lambda^{\prime}-\lambda\right)\left|t^{\prime}\right|}
\end{aligned}
$$

So

$$
2\left\|\Pi_{n}\left(f(t)-f\left(t^{\prime}\right)\right)\right\| \geq \lambda d\left(t, t^{\prime}\right)+\left(\lambda^{\prime}-\lambda\right)\left|t^{\prime}\right| .
$$

On the other hand

$$
\begin{aligned}
& \Pi_{n+1}\left(f(t)-f\left(t^{\prime}\right)\right)=T_{n+1}\left((1-\lambda) \varphi_{n+1}(t)-\left(1-\lambda^{\prime}\right) \varphi_{n+1}\left(t^{\prime}\right)\right) \text { and } \\
& {\left[(1-\lambda) \varphi_{n+1}(t)-\left(1-\lambda^{\prime}\right) \varphi_{n+1}\left(t^{\prime}\right)\right]_{\left.\right|_{s=t^{\prime}}}=(1-\lambda) d\left(t, t^{\prime}\right)+\left(\lambda-\lambda^{\prime}\right)\left|t^{\prime}\right| .}
\end{aligned}
$$

So

Therefore

$$
2\left\|\Pi_{n+1}\left(f(t)-f\left(t^{\prime}\right)\right)\right\| \geq(1-\lambda) d\left(t, t^{\prime}\right)+\left(\lambda-\lambda^{\prime}\right)\left|t^{\prime}\right| .
$$

$$
16\left\|f(t)-f\left(t^{\prime}\right)\right\| \geq d\left(t, t^{\prime}\right) .
$$

2) $2^{n} \leq|t|<2^{n+1} \leq\left|t^{\prime}\right|<2^{n+2}$, for some $n \geq 0$.

$$
\begin{gathered}
2\left\|\Pi_{n}\left(f(t)-f\left(t^{\prime}\right)\right)\right\|=2 \lambda\left\|T_{n}\left(\varphi_{n}(t)\right)\right\| \geq \lambda|t|, \\
2\left\|\Pi_{n+2}\left(f(t)-f\left(t^{\prime}\right)\right)\right\|=2\left(1-\lambda^{\prime}\right)\left\|T_{n+2}\left(\varphi_{n+2}\left(t^{\prime}\right)\right)\right\| \geq\left(1-\lambda^{\prime}\right)\left|t^{\prime}\right|, \\
\Pi_{n+1}\left(f(t)-f\left(t^{\prime}\right)\right)=T_{n+1}\left((1-\lambda) \varphi_{n+1}(t)-\lambda^{\prime} \varphi_{n+1}\left(t^{\prime}\right)\right) \text { and } \\
{\left[\lambda^{\prime} \varphi_{n+1}\left(t^{\prime}\right)-(1-\lambda) \varphi_{n+1}(t)\right]_{s=t}=\lambda^{\prime} d\left(t, t^{\prime}\right)-\lambda^{\prime}|t|+(1-\lambda)|t| .}
\end{gathered}
$$

So

$$
2\left\|\Pi_{n+1}\left(f(t)-f\left(t^{\prime}\right)\right)\right\| \geq \lambda^{\prime} d\left(t, t^{\prime}\right)-\lambda^{\prime}|t|+(1-\lambda)|t| .
$$

Combining our three estimates, we obtain

$$
24\left\|f(t)-f\left(t^{\prime}\right)\right\| \geq \lambda^{\prime} d\left(t, t^{\prime}\right)+\left(1-\lambda^{\prime}\right)\left(|t|+\left|t^{\prime}\right|\right) \geq d\left(t, t^{\prime}\right) .
$$

3) $2^{n} \leq|t|<2^{n+1}<2^{p} \leq\left|t^{\prime}\right|<2^{p+1}$ for some integers $n$ and $p$.

$$
\begin{gathered}
2\left\|\Pi_{p}\left(f(t)-f\left(t^{\prime}\right)\right)\right\|=2 \lambda^{\prime}\left\|T_{p}\left(\varphi_{p}\left(t^{\prime}\right)\right)\right\| \geq \lambda^{\prime}\left|t^{\prime}\right| \text { and } \\
2\left\|\Pi_{p+1}\left(f(t)-f\left(t^{\prime}\right)\right)\right\|=2\left(1-\lambda^{\prime}\right)\left\|T_{p+1}\left(\varphi_{p+1}\left(t^{\prime}\right)\right)\right\| \geq\left(1-\lambda^{\prime}\right)\left|t^{\prime}\right| .
\end{gathered}
$$


So

$$
24\left\|f(t)-f\left(t^{\prime}\right)\right\| \geq \frac{3}{2}\left|t^{\prime}\right| \geq\left|t^{\prime}\right|+|t| \geq d\left(t, t^{\prime}\right) .
$$

All possible cases are settled, and we have shown that $f^{-1}$ is 24 -Lipschitz.

We conclude by mentioning the following easy fact.

Proposition 2.4. Let $N$ be a metric space. The following assertions are equivalent:

(i) For any locally finite metric space $M, M \hookrightarrow N$.

(ii) There exists $C \geq 1$ such that for any locally finite metric space $M \stackrel{C}{\hookrightarrow} N$.

This is an immediate consequence of the following lemma.

Lemma 2.5. Let $\left(M_{p}, d_{p}\right)_{p=1}^{\infty}$ be a sequence of locally finite metric spaces. Then there exists a locally finite metric space $(M, d)$ such that each $M_{p}$ embeds isometrically into $M$.

Proof. For any $p \in \mathbb{N}$, pick $x_{0}^{p} \in M_{p}$. Consider $M=\bigcup_{p=1}^{\infty}\{p\} \times M_{p}$. Let $x \in M_{p}$ and $y \in M_{q}$. We define $d((p, x),(q, y))=d_{p}(x, y)$ if $p=q$ and $d((p, x),(q, y))=$ $\max \left\{p, q, d_{p}\left(x_{0}^{p}, x\right), d\left(x_{0}^{q}, y\right)\right\}$ if $p \neq q$. We leave it to the reader to check that $(M, d)$ is a locally finite metric space.

Remark 2.6. We do not know if the converse of Theorem 2.1 is true. So the question is: if every locally finite metric space metrically embeds in a given Banach space $X$, do we have that $X$ uniformly contains the $\ell_{\infty}^{n}$ 's? However, it follows from the work of Mendel and Naor in [7] that such a Banach space cannot be K-convex, or equivalently, it must contain the $\ell_{1}^{n}$ 's uniformly.

\section{REFERENCES}

[1] I. Aharoni, Every separable metric space is Lipschitz equivalent to a subset of $c_{0}^{+}$, Israel J. Math., 19 1974, 284-291. MR0511661 (58:23471a)

[2] N. Brown and E. Guentner, Uniform embeddings of bounded geometry spaces into reflexive Banach spaces, Proc. Amer. Math. Soc., 133(7) 2005, 2045-2050. MR2137870 (2006b:57044)

[3] N.J. Kalton, Coarse and uniform embeddings into reflexive spaces, preprint.

[4] G. Kasparov and G. Yu, The coarse Novikov conjecture and uniform convexity, Advances Math., to appear.

[5] J. Lindenstrauss and L. Tzafriri, Classical Banach spaces I, Springer, Berlin 1977. MR0500056 (58:17766)

[6] B. Maurey, G. Pisier, Séries de variables aléatoires vectorielles indépendantes et propriétés géométriques des espaces de Banach, Studia Math., 58(1) 1976, 45-90. MR0443015 (56:1388)

[7] M. Mendel, A. Naor, Metric cotype, preprint.

Laboratoire de Mathématiques UMR 6623, Université de Franche-Comté, 16 route de Gray, 25030 Besançon Cedex, France

E-mail address: florent.baudier@univ-fcomte.fr

Laboratoire de Mathématiques UMR 6623, Université de Franche-Comté, 16 route de Gray, 25030 Besançon Cedex, France

E-mail address: gilles.lancien@univ-fcomte.fr 